\title{
Correction to: Biogenic PLGA-zinc oxide nanocomposite as versatile tool for enhanced photocatalytic and antibacterial activity
}

\author{
Ankush Parmar ${ }^{1} \cdot$ Gurpreet Kaur $^{1} \cdot$ Shikha Kapil $^{2} \cdot$ Vipasha Sharma $^{2} \cdot$ Rajat Sandhir $^{3} \cdot$ Shweta Sharma ${ }^{1}$
}

Published online: 27 July 2019

○) King Abdulaziz City for Science and Technology 2019

Correction to: Applied Nanoscience

https://doi.org/10.1007/s13204-019-01023-3

Unfortunately, the author name (Rajat Sandhir) has been left in the online published article. The correct author group is given below:

Ankush Parmar ${ }^{1}$, Gurpreet Kaur ${ }^{1}$, Shikha Kapil ${ }^{2}$, Vipasha Sharma $^{2}$, Rajat Sandhir ${ }^{3}$, Shweta Sharma ${ }^{1, *}$

${ }^{1}$ Institute of Forensic Science and Criminology, Panjab University, Chandigarh 160014, India

${ }^{2}$ University Institute of Biotechnology, Chandigarh University, Gharuan, Punjab 140413, India

${ }^{3}$ Department of Biochemistry, Panjab University, Chandigarh 160014, India
Publisher's Note Springer Nature remains neutral with regard to jurisdictional claims in published maps and institutional affiliations.

The original article can be found online at https://doi.org/10.1007/ s13204-019-01023-3.

Shweta Sharma

25shweta@pu.ac.in

1 Institute of Forensic Science and Criminology, Panjab

University, Chandigarh 160014, India

2 University Institute of Biotechnology, Chandigarh University, Gharuan, Punjab 140413, India

3 Department of Biochemistry, Panjab University, Chandigarh 160014, India 